\title{
ANESTHESIA FOR A PATIENT WITH IMMUNE THROMBOCYTOPENIA FOR HYSTERECTOMY
}

Ravi Shankar R. B¹, Rajanna Sahukar², Anjali Rao G3, Kavyashree N. G4, Vanya Shukla5

\section{HOW TO CITE THIS ARTICLE:}

Ravi Shankar R. B, Rajanna Sahukar, Anjali Rao G, Kavyashree N. G, Vanya Shukla. "Anesthesia for a Patient with Immune Thrombocytopenia for Hysterectomy". Journal of Evolution of Medical and Dental Sciences 2014;

Vol. 3, Issue 42, September 08; Page: 10517-10522, DOI: 10.14260/jemds/2014/3371

ABSTRACT: Immune thrombocytopenia (ITP) in adults is a chronic disease resulting from increased platelet destruction and impaired platelet production. In patients with low platelet count perioperative bleeding is a major risk factor thus posing important surgical and anesthetic challenge.

KEYWORDS: Immune thrombocytopenia, neuraxial blockade, hysterectomy

INTRODUCTION: Immune thrombocytopenia is an autoimmune disorder characterized by immunologic destruction of otherwise normal patients most commonly occurring in response to unknown stimulus. It can be primary (isolated) or secondary in association with other disorders. The incidence of immune thrombocytopenia has been estimated to affect approximately 1 in 10,000 in general population about half of whom are children and account for $0.18 \%$ of hospital admission. Immune thrombocytopenia occurs most commonly in women in $2^{\text {nd }}-3^{\text {rd }}$ decade of life..$^{1,2}$

CASE REPORT: A 45yr old female patient who presented with heavy menstrual cycles for the past $10 y$ rs was planned for hysterectomy. Investigations revealed complete blood count of the patient as follows: Hb: 4.8 g/dl, Hct: 32.8\%, TC: 3440 cells $/ \mathrm{mm},^{3}$ platelet count: 0.42 lakhs $/ \mathrm{mm} .{ }^{3}$ Peripheral blood smear showed macrocytic hypochromic blood picture and thrombocytopenia .Reticulocyte count $-1.2 \%$. Bone marrow aspiration report was suggestive of Immune thrombocytopenia. Reactive status of patient was tested - HIV, HbsAg and HCV were non-reactive. Direct anti globulin test was negative. USG abdomen and pelvis showed multiple fibroids in the uterus.

She was started on oral steroid therapy with high dose dexamethasone $40 \mathrm{mg}$ daily for 4 days. 2 units of platelet and 4 units packed cells transfused. Repeat CBC count was $\mathrm{Hb}: 9.2 \mathrm{~g} / \mathrm{dl}$, platelet count 0.88 lakhs $/ \mathrm{mm},{ }^{3}$ TLC: 3440 cells $/ \mathrm{mm}^{3}{ }^{3}$ Subsequent platelet counts were done and it was noticed that there was no further drop in the platelet count. Hence she was taken up for the surgery. The platelet count on previous day of surgery was 0.90 lakhs $/ \mathrm{mm}^{3}$ and hence subarachnoid block was performed with 26 gauge spinal needle. Intraoperatively adequate hemostasis was achieved. Post-operatively the patient's vitals were stable. Repeat CBC count showed Hb- 9.4gm\%, Platelet count: 92,000 cells $/ \mathrm{mm}^{3}$ TLC: 4201 cells $/ \mathrm{mm}^{3}$.

DISCUSSION: A recent report from the international working group recommended using the term "immune" rather than "idiopathic" thrombocytopenia emphasizing the role of underlying immune mechanism. The term "purpura" was removed as many patients have no/minimal signs of bleeding at the time of diagnosis. As previously mentioned immune thrombocytopenia could be primary or secondary. ${ }^{3,4}$ 


\section{SECONDARY CAUSES:}

- autoimmune diseases (APLA)

- viral infection hepatitis C, HIV and varicella zoster

- autoimmune thrombocytopenia

- common variable immunodeficiency

- lymphoproliferative disorder

- bone marrow transplantation side effect

- systemic lupus erythematosus

- vaccination side effect

\section{The international working group also defines ITP as:}

- Newly diagnosed (diagnosis to 3 months)

- Persistent (3-12 months from diagnosis)

- Chronic (lasting for > 12 months)

\section{Basic evaluation includes:}

- Complete blood count

- Reticulocyte count

- Peripheral blood smear

- Blood group

- Direct Anti globulin Test (DAT)

- HIV

- Hepatitis C.

- Thyroid function test conducted appropriate to rule out hypothyroidism/hyperthyroidism only before elective splenectomy. ${ }^{5}$

TREATMENT: The decision to treat is based on individual patient's severity of bleeding, bleeding risk (eg. Previous bleeding episode, coincident risk factors for bleeding such as hypertension and age), activity level (eg. playing contact sports), side effects and patient preferences. Women with chronic ITP may have heavy menstrual cycles that interfere with their daily activities or result in iron deficiency anemia both of which can influence the decision to treat. Bleeding risk is increased with platelet count 20-30 x 109/L and hence considered as trigger to start treatment.3, 6

- First line treatment

- Prednisone $(1 \mathrm{mg} / \mathrm{kg} /$ day in tapering doses)

- High dose dexamethasone (40mg daily x 4 days / month for several cycles)

- Intravenous immunoglobulin $(0.8-1 \mathrm{~g} / \mathrm{kg})$

- IV anti-D Ig (50-70 micro gm/kg)

- Second line

- Rituximab (375 mg/m² weekly x 4 weeks)

- Splenectomy

- Thrombopoeitin receptor agonists 
- Romiplostim (1-10 microgm/kg) sc weekly

- Eltrombopag (25-75 mg orally daily)

- Third line

- Combination chemotherapy

- Hematopoietic stem cell transplantation

- Depending on the platelet count the following decisions can be taken

- Platelet count more than 20,000 should not be hospitalised if they are either asymptomatic or have only minor purpura.

- Platelet count more 50,000 do not require treatment - not be given glucocorticoids or IVIg as routine initial treatment.

- IVIg is also inappropriate as initial treatment with platelet counts more than 30,000 who are asymptomatic or have only minor purpura. Treatment indicated in patients with platelet counts less than 20,000 - 30,000 and those with counts less than 50,000 and significant mucosal bleeding. ${ }^{7}$

- Hospitalisation for patients with platelet count less than 20,000 who have significant mucous membrane bleeding.

- Severe life threatening bleeding patient to be hospitalised and managed in critical care unit with high dose glucocorticoid therapy, IVIg and platelet transfusion.

- Splenectomy is not to be performed as initial therapy in patients who have no bleeding, minor purpura or even mucous membrane bleeding. ${ }^{8}$

- Patient who has bleeding symptoms splenectomy is often appropriate if platelet count remains less than 30,000 after 4-6 weeks of medical treatment.

- In elective splenectomy prophylactic IVIg or oral glucocorticoid therapy with platelet count less than 20,000 .

- NOTE: Oral corticosteroids are the initial agents of choice.

- Oral prednisolone $1 \mathrm{mg} / \mathrm{kg} /$ day for 4-6 weeks is the most common initial regimen.

- High dose dexamethasone $40 \mathrm{mg}$ daily for 4 days / month for several cycles has been reported to be more effective. However in most patients the platelet count decreases once the dose is tapered or stopped; remission is sustained in only $10 \%-30 \% .{ }^{9}$

- Continuation of corticosteroids is limited by long term complication such as opportunistic infection, osteoporosis and emotional lability.

- Splenectomy remains the most effective and durable treatment in cases that are refractory to first line therapy, but its use has declined due to availability of alternative medical therapy , the associated risk of infection and concern for surgery related complications. ${ }^{10}$

\section{- Anesthetic implications:}

- Perioperative bleeding is a major anesthetic challenge. The bleeding risk increase with age and when the platelet count is less than 20-30 x 109/L. The mean platelet volume of ITP increases as the platelet count decreases and the larger platelets are assumed to be younger 
and more reactive. Hence the bleeding time (BT) of patients with thrombocytopenia is shorter than expected for the degree of thrombocytopenia and severity of bleeding in ITP is less than that seen in comparable thrombocytopenia in bone marrow failure. ${ }^{11,15}$

- Preoperative platelet count can be raised to "safe" levels by various methods. The survival time of transfused platelets is shortened to $48-230 \mathrm{~min}$ as determined by peripheral platelet count (normal half-life is 4 day). Baldim et al. concluded that there are only two major indications of platelet transfusion in patients with ITP. ${ }^{11}$

$>$ Temporary arrest of acute life threatening haemorrhage.

$>$ As preparation for surgery 1-2 hr. before performing surgery.

$>$ The increase in platelet count can be expected to last for around 1 week following IVIg infusion. Therefore IVIg is an important agent for preoperative treatment of planned surgical procedure. However treatment with IVIg may not cause an increase in platelet count until 2-3 days following initiation of treatment. Hence can be used as effective postoperative therapy.

\section{- Emergency management of ITP:}

- General measures to decrease bleeding risk including drugs that interfere with platelet count and hemostatic function, control of blood pressure and minimise risk of trauma.

- Initiated with IVIg $1 \mathrm{gm} / \mathrm{kg} /$ day for $1-3$ consecutive days or IV anti-D (75 micro gram $/ \mathrm{kg}$ ) and IV methylprednisolone1-2gm/day for 1-3 consecutive days unless platelet count is more than 30,000-50,000.

- Platelet transfusion ranging from every $30 \mathrm{~min}-8 \mathrm{hrs}$ and platelet transfusion in conjunction with continuous infusion of IVIg.

- Recombinant factor VIIa has been used in several patients with ITP who were either bleeding or undergoing surgery (care should be taken as this can cause thrombosis).Recombinant factor VIIa can be administered if bleeding remains uncontrolled while awaiting response to treatment. Dosing of minimum $40 \mathrm{U} / \mathrm{kg}$ is used successfully in patients with thrombocytopenia with diverse etiologies.

- Antifibrinolytic agents (amino caproic acid and tranexamic acid)are discussed in case reports and reviews as an adjunct treatment for bleeding in thrombocytopenia patients but the efficacy remains unproved. $3,11,14$

\section{- ITP IN PREGNANCY:}

- Immune thrombocytopenia can occur denovo with pre-existing ITP. Intrapartum and postpartum haemorrhage life threatening to mother. Platelet associated IgG (PAIgG) can cross placenta - fetus may become thrombocytopenic and is at risk haemorrhage particularly in CNS.

$>$ A platelet count $>50 \times 109 / \mathrm{L}$ should be considered safe for vaginal bleeding in patients with normal coagulation

$>$ A platelet count $>80 \times 10^{9} / \mathrm{L}$ should be considered safe for LSCS, spinal and epidural with normal coagulation

- Mode of delivery determined by obstetric consideration alone

- In the neonate platelet count may be low and continue to be low in the first week of life.12,14 
CONCLUSION: Immune thrombocytopenia is a condition characterized by thrombocytopenia to increased platelet destruction and impaired platelet production affecting patients of any age group. It could be due to primary or secondary causes and treatment should be started based on individual patient profile and platelet count. These patients who are taken for surgery pose risk of life threatening hemorrhage and major anesthetic and surgical challenge to achieve hemostasis. Various medical modalities of treatment such as corticosteroids, IVIg and chemotherapeutic agents can be used. Splenectomy has a minimal role to play as the initial mode of management. ${ }^{13}$

\section{REFERENCES:}

1. Brain-J Pollard, T Ainley. Blood: Idiopathic thrombocytopenic purpura. Handbook of Clinical Anesthesia, 2nd Edn, Churchill Livingstone, $\mathrm{p}$ 210-211.

2. Waller RW, Walker W. Idiopathic thrombocytopenia, initial illness and long term follow up. Arch Dis Child 59: 316, 1984.

3. British Committee for standards in Haematology Guidelines for investigations and management of ITP in adults, children and pregnancy. British Journal of Haematology 2003; 120: 574-596.

4. Rodeghiero F, Stasi R, Gernsheimer T. Standardization of terminology, definitions and outcome criteria in immune thrombocytopenic purpura of adults and children: report from an international working group. Blood 2009; 113: 2386-2393.

5. Gernsheimer T. Chronic idiopathic thrombocytopenic purpura: mechanisms of pathogenesis. Oncologist.2009; 14: 12-21.

6. Lacey JV, Penner JA. Management of Idiopathic thrombocytopenic purpura in adult. Semin Thromb Hemost 3: 160, 1977.

7. Duhem C, Dicato MA, Ries F. Side effects of intravenous immune-globulins. Clin Exp Immunol 97: 79, 1994 (suppl I).

8. Benham ES, Taft LI. Idiopathic thrombocytopenic purpura in children: Results of steroid therapy and splenectomy. Aust Paediat J 8: 311, 1972.

9. Anderson JC. Response of resistant idiopathic thrombocytopenic purpura and high dose dexamethasone therapy. New England Journal of Medicine 1994; 330: 1560-1564.

10. Mc Verry BA. Management of ITP in adults. British Journal of Haematology 1985; 59: 203-208.

11. Yoshitaka Toyomasu, Rinshun Shimabukuro, Hatson Moriyama, Daihiko Eguchi, Koichi Ishikawa, Fumiaki Kishihara, Yasurou Fukuyama, Takashi Matsumata, Erito Mochiki, Hiroyuki Kuwano. Successful perioperative management of a patient with idiopathic thrombocytopenic purpura undergoing emergent appendectomy. Int J Surg Case Rep.4 (10): 898-900, 2013.

12. P C A Kam, S A Thompson, A C S Liew. Thrombocytopenia in parturient. Anaesthesia 2004, 59: 225:264.

13. George JN, El-Harake MA, Aster RH. Thrombocytopenia due to enhanced platelet destruction by immunologic mechanisms in Bentler. E, Lichtman MA, Coller BS, Kipps JJ (eds). Williams Hematology. New York, INY. Mc Graw Hill, 1995, p 1315.

14. Anguilo J P, Temple J T, Corrigan J J, Jr. Management of caesarean section in a patient with idiopathic thrombocytopenic purpura. Anesthesiology. 1977; 46 (February (2)): 145-147.

15. Slicther S J. Relationship between platelet count and bleeding risk in thrombocytopenic patients. Transfusion Medicine Reviews .2004; 18 (July (3)): 153-167. 


\section{CASE REPORT}

\section{AUTHORS:}

1. Ravi Shankar R. B.

2. Rajanna Sahukar

3. Anjali Rao G.

4. Kavyashree N. G.

5. Vanya Shukla

\section{PARTICULARS OF CONTRIBUTORS:}

1. Professor, Department of Anaesthesiology, JJM Medical College, Davanagere.

2. Professor, Department of Anaesthesiology, JJM Medical College, Davanagere.

3. Post Graduate, Department of Anaesthesiology, JJM Medical College, Davanagere.

4. Post Graduate, Department of Anaesthesiology, JJM Medical College, Davanagere.
5. Post Graduate, Department of Anaesthesiology, JJM Medical College, Davanagere.

\section{NAME ADDRESS EMAIL ID OF THE CORRESPONDING AUTHOR:}

Dr. Ravi Shankar R. B, 4798, $2^{\text {nd }}$ Cross,

S. S. Layout, Davanagere-577004.

Email: rbravi2006@gmail.com

Date of Submission: 22/08/2014.

Date of Peer Review: 23/08/2014.

Date of Acceptance: 03/09/2014.

Date of Publishing: 08/09/2014. 